\title{
Determining Subcellular Localization of Selenoprotein Translation Factors using Immunofluorescence
}

Qingping He*, Lucia A. de Jesus*, Tanya Michaud*, Peter R. Hoffmann*, Robert J. Stillwell ${ }^{*}$, John W. Harney**, Marla J. Berry*

*Department of Cell and Molecular Biology, University of Hawaii at Manoa, Honolulu HI 96822

**Thyroid Division, Brigham and Women's Hospital and Harvard Medical School, Boston MA 02115

Selenoproteins exhibit a wide range of functions and are unique in that their mRNA contain UGA codons, which typically signal termination to the protein synthesis machinery. For selenoproteins, UGA is recoded to selenocysteine and this recoding requires special translation factors, including a SECIS binding protein (SBP2) and a dedicated elongation factor (EFsec). We hypothesized that these factors must bind to mRNA in the nucleus for efficient translation of selenoproteins to occur.

Using bioinformatic approaches, we identified potential nuclear localization signals (NLS) and nuclear export signals (NES) in the amino acid sequences for both SBP2 and EFsec (Fig. 1A and B). Expression plasmids were then constructed encoding full-length versions of these two proteins with various $\mathrm{N}$-terminal or $\mathrm{C}$-terminal epitope tags as well as truncated or mutated versions of the proteins with the same epitope tags. These expression plasmids were then transiently transfected into two different cell lines and immunofluorescence used to determine the subcellular localization of each form of SBP2 and EFsec.

Full-length versions of both EFsec and SBP2 were found to be predominantly cyptoplasmic when transiently expressed in either MSTO cells or HEK293T cells (Fig. $1 \mathrm{C}$ and $\mathrm{F}$ ). When a truncated version of EFsec was expressed lacking the NES, this protein was localized to the nucleus (Fig. 1D). This supported the notion that the protein containing the NLS was able to be imported into the nucleus and accumulate there because of the deletion of the NES. This was further supported when the same truncated protein lacking the NES but containing mutations (lysine $\rightarrow$ alanine) in the NLS was found to revert from nuclear back to cytoplasmic localization (Fig. 1E). Similarly, different truncated versions of SBP2 lacking most or all of the NES were found to be localized to the nucleus, suggesting that one or more of the N-terminal NES are functional in export from the nucleus, and that at least one NLS in the 399-517 region as well as the NLS in the 517-777 region are functional in protein import.

Taken together, these results suggest that both EFsec and SBP2 contain functional NLS and NES. Furthermore, import and export of these proteins may play a crucial role in assembly with selenoprotein mRNA and recoding of the UGA codon during protein translation. 
A

EFsec

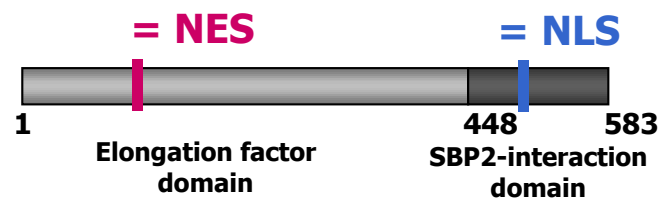

$\mathrm{C}$

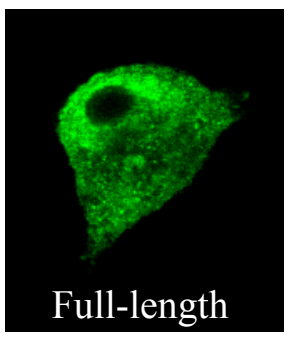

$\mathrm{D}$

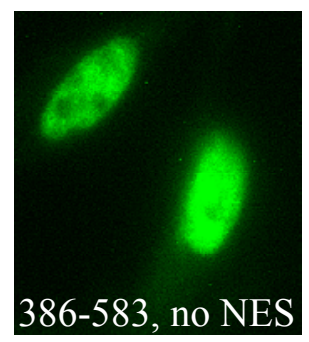

E

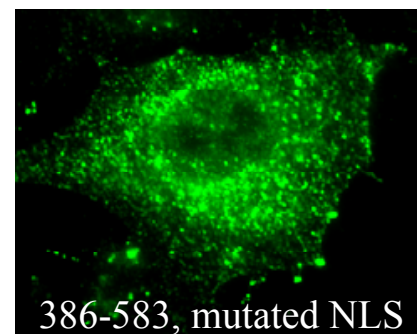

B

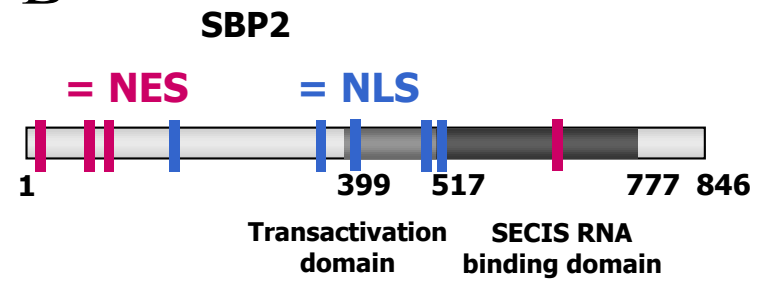

$\mathrm{F}$
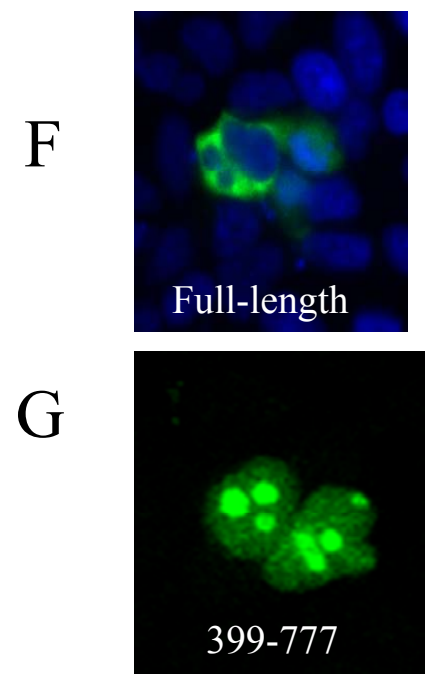

$\mathrm{H}$
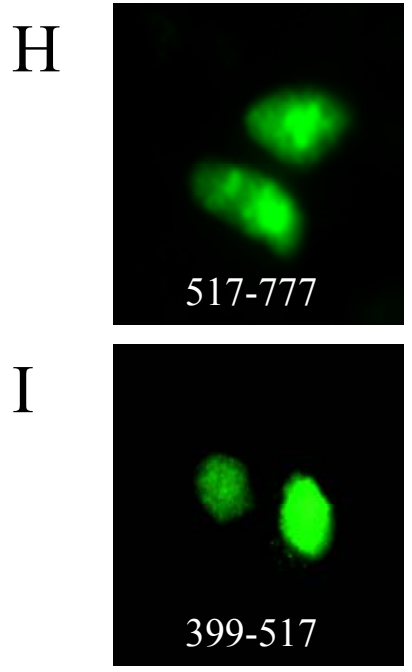

Figure 1. Primary structure models of EFsec (A) and SPB2 (B) with NLS (blue) and NES (pink) domains highlighted. Subcellular localization of FLAG-tagged, full-length (C), truncated (D), or truncated, mutated (E) EFsec. Subcellular localization of V5-tagged full-length (F) or truncated (G-I) SBP2. Green fluorescence (Alexa-488) indicates protein and blue fluorescence (DAPI) indicates nuclear staining. 